\title{
NORMAL CLASSES OF PRIME RINGS DETERMINED BY MODULES ${ }^{1}$
}

\author{
W. K. NICHOLSON AND J. F. WATTERS
}

\begin{abstract}
It is shown that primitive rings with a socle, left weakly primitive rings and prime Johnson rings constitute normal classes of rings. The technique involves characterizing each class as all rings with a faithful module of a certain type.
\end{abstract}

1. Introduction. A Morita context $(R, V, W, S)$ consists of two rings $R$ and $S$ and two bimodules ${ }_{R} V_{S}$ and ${ }_{S} W_{R}$ together with mappings $V \times W \rightarrow R$ and $W \times V \rightarrow$ $S$ (written multiplicatively) which induce bimodule homomorphisms $V \otimes_{S} W \rightarrow R$ and $W \otimes_{R} V \rightarrow S$ and which satisfy the associativity conditions

$$
(v w) v^{\prime}=v\left(w v^{\prime}\right) \text { and }(w v) w^{\prime}=w\left(v w^{\prime}\right)
$$

for all $v, v^{\prime}$ in $V$ and $w, w^{\prime}$ in $W$. See Amitsur [1] for details. An equivalent formulation is that $\left[\begin{array}{ll}R & V \\ W & S\end{array}\right]$ is a ring with the usual matrix operations. An important example is the standard context $\left(R, M, M^{*}, E\right)$ determined by an $R$-module $M$ where $E=\operatorname{end}_{R} M$. We call a Morita context $(R, V, W, S) S$-faithful if $S \neq 0$ and $V s W=0$ implies $s=0$ for $s \in S$. Then a normal class $\mathcal{P}$ is defined [3] to be a class of prime rings such that if $(R, V, W, S)$ is an $S$-faithful context with $R \in \mathscr{P}$ then necessarily $S \in \mathcal{P}$. These normal classes enjoy many pleasant properties and the reader is referred to [3] for details. Examples of normal classes include the classes of prime and left primitive rings [1] and it is the intention here to show that the class of primitive rings with a nonzero socle is normal as is the class of prime nonsingular rings with uniform left ideal. Rowen [5] calls these prime Johnson rings and has characterized them as essential prime subrings of primitive rings with nonzero socle.

As well as these, two more classes of rings are of interest to us here. Zelmanowitz [7] has called a ring left weakly primitive if it has a faithful, monoform, compressible left module and has proved a version of the Jacobson Density Theorem for these rings. Finally, Ortiz [4] has introduced the concept of a $K$-primitive ring which we will redefine later.

The principal aim of this note is to prove the following

THEOREM. The following classes of prime rings are normal:

(1) the primitive rings with nonzero socle,

Received by the editors June 25, 1980.

AMS (MOS) subject classifications (1970). Primary 16A12, 16A20; Secondary 16A21.

$K e y$ words and phrases. Normal class, primitive ring, weakly primitive ring, Johnson ring, $K$-primitive ring, normal radical.

${ }^{1}$ This research was partially supported by N.S.E.R.C. Grant \#A8075.

(C) 1981 American Mathematical Society 0002-9939/81/0000-0406/\$02.00 
(2) the left weakly primitive rings,

(3) the prime Johnson rings,

(4) the K-primitive rings.

2. Module properties and Morita contexts. Throughout this paper, unless otherwise mentioned, rings need not have a unity, modules are left modules, and homomorphisms are written on the right. If $R$ is a ring, then $R^{1}$ denotes $R$ if $R$ has a unity and, if not, it denotes the usual ring with unity containing $R$ as an ideal. If $M$ is a module and $X \subseteq M$ the left annihilator of $X$ will be written $l(X)$. All classes of rings and modules are assumed to be nonempty and closed under isomorphisms.

The classes of modules to be considered consist of prime modules. Here a module $M$ is called prime if $R M \neq 0$ and, whenever $A m=0$ where $m \in M$ and $A$ is an ideal of $R$, then either $A M=0$ or $m=0$. Another notion of prime module was introduced by Zelmanowitz [6] and, to distinguish this from the above concept, we refer to these as standard prime. Thus $M$ is standard prime if $M \neq 0$ and $m_{1} M^{*} m_{2}=0, m_{i} \in M$, implies $m_{1}=0$ or $m_{2}=0$ where $M^{*}=\operatorname{Hom}_{R}(M, R)$. Observe that a ring $R$ is prime if and only if ${ }_{R} R$ is prime and faithful if and only if ${ }_{R} R$ is standard prime and faithful.

A module $M$ is called compressible if $R M \neq 0$ and $M$ can be embedded in each nonzero submodule. Note that all compressible and standard prime modules are prime and that each of these classes of modules is closed under taking nonzero submodules.

A module $M$ is called monoform if every partial endomorphism $\alpha: N \rightarrow M$, $N \subseteq M$, is either zero or monic. Such modules are uniform and we shall use the fact that a uniform, nonsingular module is necessarily monoform. We use the term nonsingular to mean that the singular submodule $Z(M)$ is zero. Here $Z(M)=\{m$ $\in M \mid l(m)$ is essential in $R\}$.

We shall define a module ${ }_{R} M$ to have property $K$ if $M$ is prime and $l(M / N)=$ $l(M)$ for ${ }_{R} N \subseteq{ }_{R} M$ only when $N=0$. Then a $K$-primitive ring [4] is a ring with a faithful module having property $K$.

Proposition. Let $(R, V, W, S)$ be a Morita context and let $M=R^{1} m$ be a left $R$-module such that $(V S W) M \neq 0$. Put

$$
U=\{w \in W \mid V w m=0\} .
$$

If ${ }_{R} M$ has any of the following properties, so also does the $S$-module $W / U$ :

(1) irreducible,

(2) prime,

(3) compressible,

(4) prime and monoform.

If in addition $(R, V, W, S)$ is $S$-faithful we can add:

(5) faithful,

(6) standard prime,

(7) property $K$.

Proof. (1) and (2) are proved as in Amitsur [1] and (5) is an easy consequence of the definitions. The proof of (3) is straightforward and is left to the reader. 
(4) $W / U$ is prime by (2). Suppose $0 \neq \alpha: W_{0} / U \rightarrow W / U$ is $S$-linear where $W_{0}$ is an $S$-submodule of $W$. We must show $\alpha$ is monic. Let $\left(w_{0}+U\right) \alpha=w_{1}+U \neq 0$ and define $\alpha_{1}: V w_{0} m \rightarrow M$ by $\left(v w_{0} m\right) \alpha_{1}=v w_{1} m$. If it happens that $v w_{0} m=0$, then $V W v w_{1} m=0$ so $v w_{1} m=0$ because $M$ is prime. Hence $\alpha_{1}$ is well defined and nonzero so it is monic by hypothesis. We show that this implies $\alpha$ is monic. Suppose, if possible, that $\left(w_{2}+U\right) \alpha=0$ where $w_{2} \in W_{0}-U$. Then $V w_{2} m \neq 0$ so, since $M$ is uniform (being monoform), we have $V w_{2} m \cap V w_{0} m \neq 0$, say $v_{2} w_{2} m=v_{0} w_{0} m \neq 0$. Then $W\left(v_{2} w_{2}-v_{0} w_{0}\right) \subseteq U$ so, for all $w \in W$,

$$
0=w v_{2}\left(w_{2}+U\right) \alpha=\left(w v_{0} w_{0}+U\right) \alpha=w v_{0} w_{1}+U \text {. }
$$

This means $V W v_{0} w_{1} m=0$ so $0=v_{0} w_{1} m=\left(v_{0} w_{0} m\right) \alpha_{1}$, a contradiction. Hence $\alpha$ is monic as required.

(6) Assume $M$ is standard prime. If $w_{1}+U$ and $w_{2}+U$ are nonzero in $W / U$, let $v_{1} w_{1} m \neq 0 \neq v_{2} w_{2} m, v_{i} \in V$. By hypothesis there exists $\lambda: M \rightarrow R$ such that $\left[\left(v_{1} w_{1} m\right) \lambda\right]\left(v_{2} w_{2} m\right) \neq 0$. Define $\gamma: W / U \rightarrow S$ by $(w+U) \gamma=w(m \lambda) v_{2}$. If $w \in U$ then $V w m=0$ so $V w(m \lambda) v_{2} W=0$. This implies $w(m \lambda) v_{2}=0$ (since the context is $S$-faithful) so $\gamma$ is well defined. It remains to show that $\left(w_{1}+U\right) \gamma\left(w_{2}+U\right) \neq 0$. But if this equals 0 then $V w_{1}(m \lambda) v_{2} w_{2} m=0$, contrary to the choice of $\lambda$.

(7) Let $W \supseteq{ }_{s} T \supset U$ and suppose that $l(W / T)=l(W / U)$. Pick $t \in T-U$. Then $V t m \neq 0$ and $\exists a \in R$ such that $0 \neq a M \subseteq V t m$ by property $K$. Since $M$ is prime $l(M)$ is a prime ideal of $R$ so $a \notin l(M)$ and $V W \nsubseteq l(M)$ imply that $V W a V W \nsubseteq l(M)$ and so $\exists b \in W a V$ with $V b W \nsubseteq l(M)$. Hence $V b W m \neq 0, b W$ $\nsubseteq U$

On the other hand $a V W M \subseteq a M \subseteq V$ tm, so given $v \in V, w \in W \exists v^{\prime} \in V$ with $a v w m=v^{\prime} t m$. Hence $V W\left(a v w-v^{\prime} t\right) m=0, W\left(a v w-v^{\prime} t\right) \subseteq U$, and $W a v w \subseteq T$. Thus $W a V \subseteq l(W / T)=l(W / U)$ so $W a V W \subseteq U$. In particular $b W \subseteq U$ giving a contradiction. Therefore $W / U$ has property $K$.

3. Proof of Theorem. In each of the first three cases we characterize the rings considered as those having a faithful module of some specific type.

(1) If $R$ is primitive with nonzero socle and $L$ is a minimal left ideal of $R$, then $L$ is a faithful, standard prime, irreducible $R$-module. Conversely, if $M$ is a faithful, standard prime, irreducible module and $0 \neq \alpha \in M^{*}$, then $M \cong M \alpha$ which is a minimal left ideal of the prime ring $R$. Hence $R$ is primitive with nonzero socle. The normality of this class now follows from (1), (5) and (6) of the Proposition.

(2) Since compressible modules are prime, this is immediate from the definitions and parts (3), (4) and (5) of the Proposition.

(3) If $R$ is a prime Johnson ring and $L$ is a nonsingular uniform left ideal of $R$, then $L$ is a faithful, standard prime, monoform module. For the converse suppose that $M$ is a faithful, standard prime, monoform module. Let $0 \neq \alpha \in M^{*}$. Then $(M \alpha)^{2} \neq 0$ so let $m^{\prime} \alpha m \neq 0$ where $m^{\prime}, m \in M$. The map $\alpha m: M \rightarrow M$ is monic by hypothesis so $M \cong M \alpha \subseteq R$. Suppose now that $b \in Z(M \alpha)$. Then $M \alpha \cap l(b) \neq 0$ so the map $\beta: x \mapsto x b$ is not monic on $M \alpha$. Hence $\beta=0$ so $M \alpha \subseteq l(b)$. But then $M \alpha Z(M \alpha)=0$ whence $Z(M \alpha)^{2}=0$. Thus $Z(M \alpha)=0$ since $R$ is a prime ring. Using this characterization of prime Johnson rings the normality follows from (4), (5) and (6) of the Proposition. 
(4) Let ${ }_{R} M$ have property $K$. We are done if the property $K$ is inherited by nonzero submodules, for then we can take $M=R^{1} m$ and the normality follows from (5) and (7) of the Proposition. Suppose $0 \neq{ }_{R} N \subseteq M$. Then $l(N)=l(M)$. If ${ }_{R} K \subseteq N$ and $l(N / K)=l(N)$, then $l(M / K) \subseteq l(N / K)=l(N)=l(M) \subseteq$ $l(M / K)$, so $K=0$ and $N$ has property $K$ as required.

Bergman has given an example of a ring primitive on one side but not on the other but the analogous question for weakly primitive rings is still open. Of course the notion of a primitive ring with a socle is left-right symmetric but the following example shows that this is not true of the prime Johnson rings.

EXAMPLE. Let $F$ be a field with a monomorphism $\alpha: F \rightarrow F$ which is not onto. Then the skew polynomial ring $R=F[x, \alpha]$ is prime, left and right nonsingular, and has a uniform left ideal but no uniform right ideal. In fact, the prime and nonsingularity conditions are clear since $R$ is a domain, and ${ }_{R} R$ is uniform since $R$ is left Öre. However, if $T$ is a uniform right ideal and $0 \neq b \in T$, the map $r \mapsto b r$ is an embedding $R \rightarrow T$. This implies $R$ is right Öre, a contradiction.

REMARK. If $\mathcal{P}$ is any normal class of prime rings, we may define a radical in the category of rings by

$$
N_{\mathscr{P}}(R)=\bigcap\{P \mid P \text { is an ideal of } R \text { and } R / P \in \mathcal{P}\} .
$$

Then $N_{\mathscr{P}}$ is a normal and special radical (see [3]) and was considered in [1] when $\mathscr{P}$ is the class of left primitive rings or the class of prime rings. If $\mathcal{P}$ consists of the left weakly primitive rings then $N_{\mathscr{P}}$ is the weak radical [2] while if $\mathscr{P}$ is the class of $K$-primitive rings, the radical is discussed in [4]. Properties of these radicals (e.g. $N_{\mathscr{P}}\left[M_{n}(R)\right]=M_{n}\left[N_{\mathscr{P}}(R)\right]$; see [2] and [4]) now follow easily from general results in [3].

Call a left ideal $L$ in a ring $R$ full if $r R \subseteq L, r \in R$, implies $r \in L$. Suppose $\Re$ is a class of prime modules giving rise, as in the theorem, to a normal class

$$
\mathscr{P}=\{R \mid R \text { has a faithful module in } \mathfrak{R}\} \text {. }
$$

Then the radical $N_{\mathscr{P}}$ above has the following description

$$
N_{\mathscr{P}}(R)=\bigcap\{L \mid L \text { is a full left ideal of } R \text { and } R / L \in \mathfrak{T}\} .
$$

The verification is omitted.

\section{REFERENCES}

1. S. A. Amitsur, Rings of quotients and Morita contexts, J. Algebra 17 (1971), 273-298.

2. K. Koh and A. C. Mewborn, The weak radical of a ring, Proc. Amer. Math. Soc. 18 (1967), 554-559.

3. W. K. Nicholson and J. F. Watters, Normal radicals and normal classes of rings, J. Algebra 59 (1979), 5-15.

4. A. H. Ortiz, On the structure of semiprime rings, Proc. Amer. Math. Soc. 38 (1973), 22-26.

5. L. Rowen, Monomial conditions on prime rings, Israel J. Math. 27 (1977), 131-149.

6. J. Zelmanowitz, Semiprime modules with maximum conditions, J. Algebra 25 (1973), 554-574.

7. __ Dense rings of linear transformations, Ring Theory II, Second Oklahoma Conference (McDonald-Morris, Ed.), Dekker, New York, 1977, pp. 281-295.

Department of Mathematics and Statistics, University of Calgary, Calgary t2N 1N4, CANADA

DePartment of Mathematics, University OF Leicester, Leicester LE1 7RH, ENGLAND 